\title{
Type 1 neurofibromatosis in the practice of a dermatologist: a case report
}

\section{Igor Kuklin', Nikolay Kungurov1, Natalia Zilberberg', Muza Kokhan', Galina Safonova1, Nina Toropova1, Maria Kuklina ${ }^{2}$}

${ }^{1}$ Ural Research Institute of Dermatovenereology and Immunopathology, Ekaterinburg, Russia, ${ }^{2}$ Ural Federal University named after the first President of Russia B.N. Yeltsin, Ekaterinburg, Russia

Corresponding author: Dr. Igor Kuklin, E-mail: kuklin71@mail.ru

\begin{abstract}
Dermatologists can be a specialist of the "first contact" when patients have skin manifestations, simulating chronic dermatoses. A 27-year-old patient presented at our Institute with multiple tumor-like lesions accompanied by moderate itching. Based on the medical history, clinical and pathomorphological data, the patient was diagnosed with type I neurofibromatosis (von Recklinghausen disease). This clinical case demonstrates a late diagnosis of a relatively rare disease - type I neurofibromatosis, when the patient was left without monitoring by pediatricians, dermatologists and neuropathologists for a long time.
\end{abstract}

Key words: Type I neurofibromatosis; Diagnosis; Clinical manifestations

\section{INTRODUCTION}

Dermatologists often encounter diseases, simulating chronic dermatoses, in their practice. The significance of the problem of timely and correct diagnosis of these diseases is determined by the fact that a dermatologist, as a rule, is a specialist of the "first contact" when a patient with exophytic tumour skin manifestations seeks medical advice [1-6].

In order to attract the attention of dermatologists to the problem of timely diagnosis of diseases simulating chronic dermatoses, we describe a clinical case of late diagnosis of neurofibromatosis in a young man aged 27.

\section{CASE REPORT}

Patient B., born in 1991, presented at the clinic of the Ural Research Institute of Dermatovenerology and Immunopathology with complaints for disseminated rash on the skin of the trunk, upper and lower extremities, accompanied by moderate itching.
Anamnesis morbi: B. considered himself sick for 2 years, when he first paid attention to the appearance of a large number of tumor-like lesions on the skin of the abdomen and back, not accompanied by subjective sensations. However, with additional questioning, the first spots on the abdominal skin were found to be noted at the age of 4 , but then the parents mistakenly associated them with the consequences of thermal burns in the child.

Anamnesis vitae and occupational history of the patient were unremarkable. There were no skin diseases or onychopathology in the family history.

The patient's general condition was satisfactory. The breathing was vesicular, without wheezing. The heart sounds were clear and rhythmic. The abdomen was soft and painless on palpation. The liver was at the costal arch. The bowel and bladder functioned normally.

The unaffected skin areas were of physiological colour, normal temperature, humidity and turgor. The oral cavity mucosa was of physiological colour and free

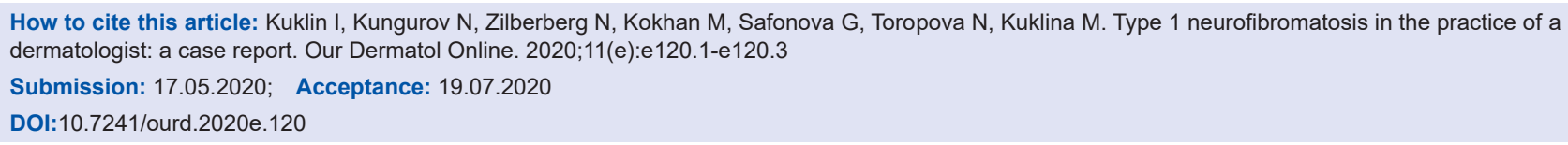


from rashes. The skin involvement was disseminated. Elements were located on the skin of the trunk and upper extremities, presented as pigmented spots of the colour of coffee with milk (café-au-lait spots), with a smooth surface and clear borders, oval, $1.5 \times 4.5 \mathrm{~cm}$ in size. Numerous subcutaneous tumor-like lesions of a rounded shape, soft-elastic consistency, up to $2.0 \mathrm{~cm}$ in diameter were located over the entire surface of the trunk and upper limbs. The skin of the axillary regions had numerous small pigmented spots up to $2-3 \mathrm{~mm}$ in diameter, light brown in color (axillary freckling known as the Crowe's sign). The skin of the abdomen, back and left lateral surface of the neck was marked by nodes of 2.5 to $5.5 \mathrm{~cm}$ in diameter, soft elastic consistency, flesh-coloured or light brown, and painless on palpation (Figs. 1 and 2). Hair and nails were unchanged. Dermographism was red and persistent.

The complete blood count and urinalysis were unremarkable. The serological Treponema pallidum test was negative. HIV and hepatitis $\mathrm{B}$ or $\mathrm{C}$ antibodies were not detected. There were no deviations in the biochemical blood test.

The council of physicians of the Ural Research Institute of Dermatovenereology and Immunopathology suspected neurofibromatosis (von Recklinghausen's disease) for the first time in the patient, and an incisional skin biopsy of the most infiltrated tumour element was performed.

The results of the pathomorphological study of skin biopsy showed epidermis without significant changes. The tumour node was localized in the dermis, separated from the epidermis by a narrow strip of unchanged collagen, presented by randomly oriented fibrous structures and cellular elements, mainly fibroblasts and lemmocytes (Schwann cells) with an admixture of mast cells, granulocytes and leukocytes (Fig. 3).

Based on the medical history, clinical and pathomorphological data, the patient was diagnosed with type I neurofibromatosis, and symptomatic therapy was prescribed, including Ketotifen 1 mg twice daily orally and Advantan emulsion (Methylprednisolone Aceponate) twice daily topically on the elements. The patient was discharged from the hospital and advised to visit a neurologist, cardiologist, ophthalmologist and nephrologist for additional in-depth examinations to clarify the pathology of visceral organs associated with the underlying disease.

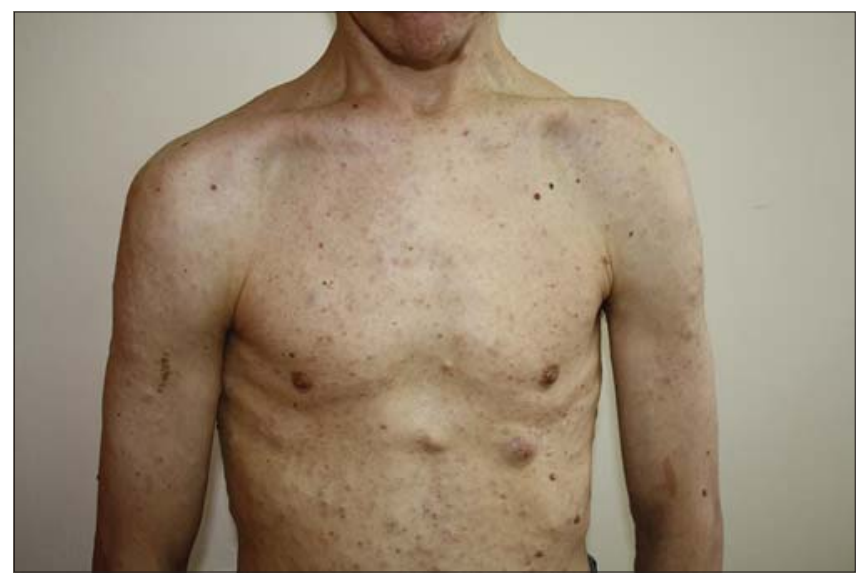

Figure 1: Patient B., 27 years old, pigmented café-au-lait spots and multiple neurofibromas on the skin of his thorax and abdomen.

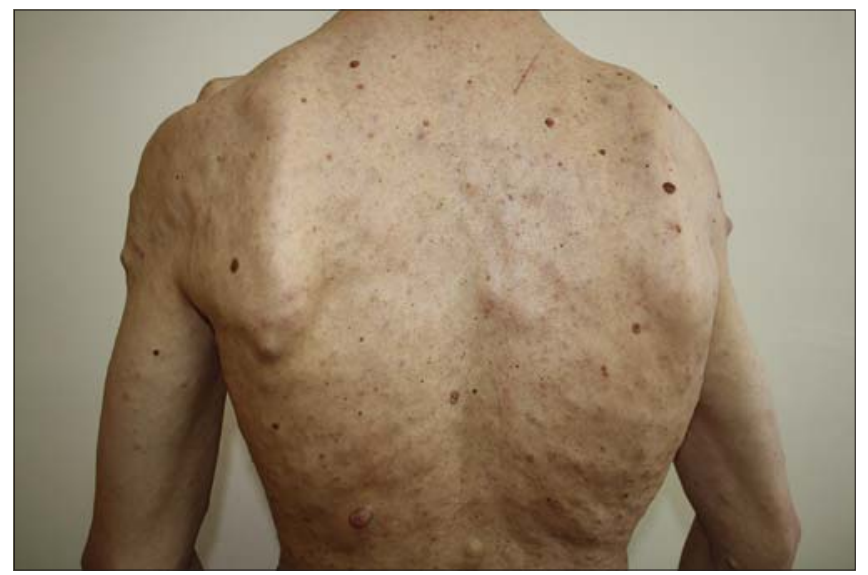

Figure 2: The same patients, multiple neurofibromas on the skin of his back.

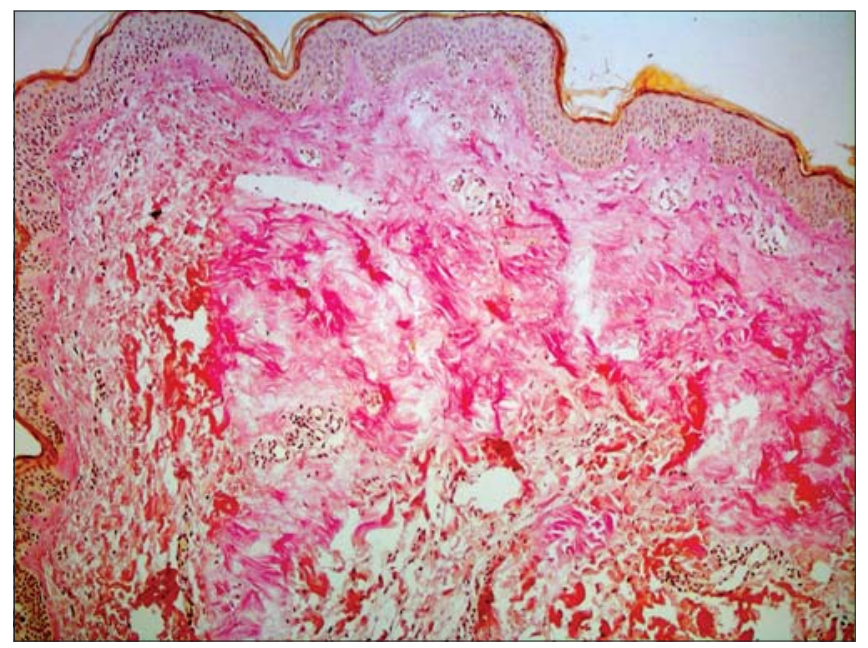

Figure 3: Patient B., a pathomorphological study of skin biopsy (Van Gieson staining at $\times 100$ magnification).

\section{DISCUSSION}

The presented clinical case deserves attention due to the fact that a patient with neurofibromatosis has remained for a long time out of sight of pediatricians, 
dermatologists and neuropathologists. A thorough clinical examination, a carefully collected medical history, and a detailed examination of the patient with a pathological study of skin biopsy are the prerequisites for the timely diagnosis of the disease.

\section{Consent}

The authors certify that they have obtained all appropriate patient consent forms. In the form the patient has given his consent for his images and other clinical information to be reported in the journal. The patient understand that his name and initials will not be published and due efforts will be made to conceal their identity.

\section{REFERENCES}

1. Micieli R, Salsberg JM. Visual dermatology: mosaic generalized neurofibromatosis 1. J Cutan Med Surg. 2019;23:328.
2. Tanito K, Ota A, Kamide R, Nakagawa H, Niimura M. Clinical features of 58 Japanese patients with mosaic neurofibromatosis 1. J Dermatol. 2014;41:724-8.

3. Miraglia E, Calvieri S, Giustini S. Pruritus in neurofibromatosis type 1. G Ital Dermatol Venereol. 2018;153:120-2.

4. Yao R, Wang L, Yu Y, Wang J, Shen Y. Diagnostic value of multiple café-au-lait macules for neurofibromatosis 1 in Chinese children. J Dermatol. 2016;43:537-42.

5. Reschke R, Kadlecova M, Grunewald S, Ziemer M. Rare form of a segmental neurofibromatosis with giant plexiform neurofibroma. Hautarzt. 2019;70:638-40.

6. Slesarenko NA, Morrison AV, Marchenko VM, Moiseev AA, Sherstobitova KYu, Ishmukhametova RA. Neurofibromatosis in the practice of a dermatologist. Saratov Scien Med J. 2018;14:760-3.

Copyright by Igor Kuklin, et al. This is an open access article distributed under the terms of the Creative Commons Attribution License, which permits unrestricted use, distribution, and reproduction in any medium, provided the original author and source are credited.

Source of Support: Nil, Conflict of Interest: None declared. 